\title{
DONOR ANTIGEN-PRESENTING CELLS ARE IMPORTANT IN THE DEVELOPMENT OF OBLITERATIVE AIRWAY DISEASE
}

Wilson Y. Szeto, MD

Alyssa M. Krasinskas, MD

Daniel Kreisel, MD

Sicco H. Popma, BS ${ }^{\mathrm{a}}$

Bruce R. Rosengard, MD
Objective: Obliterative airway disease, which resembles obliterative bronchiolitis histologically, develops in murine heterotopic tracheal allografts. Chimeric tracheas were used to examine whether donor-type antigen-presenting cells are important in the development of obliterative airway disease. To separate the contributions of $\mathrm{CD}^{+}$and $\mathrm{CD}^{+}$direct pathways, we transplanted tracheas from knockout mice lacking major histocompatibility complex (MHC) class I or II antigens.

Methods: Chimeric tracheas were created via bone marrow transplantation in fully MHC-mismatched combinations. Tracheas from naive B6, autologously reconstituted $\mathrm{B} 6$, chimeric $\mathrm{B} 6$ bearing recipient-type $\mathrm{C} 3 \mathrm{H}$ antigen-presenting cells, MHC class I knockout B6 (B6 $\left.{ }^{\mathrm{I}}\right)$, MHC class II knockout B6 $\left(\mathrm{B} 6^{\mathrm{II}-}\right)$, or $\mathrm{C} 3 \mathrm{H}$ mice were transplanted into $\mathrm{C} 3 \mathrm{H}$ recipients. The tracheas were harvested at days 14 and 28.

Results: At day 28, isografts showed no occlusion, normal respiratory epithelium, and minimal infiltrates. Naive or autologously reconstituted B6, B6 ${ }^{\mathrm{I}-}$, and $\mathrm{B} 6^{\mathrm{II}}{ }^{-}$tracheas showed minimal occlusion at day 14 but contained intraepithelial infiltrates. By day 28 , the naive or autologously reconstituted B6 tracheas had occlusion of $69.5 \% \pm 11.6 \%$ (mean \pm standard error of the mean), and in comparison, $\mathrm{B} 6^{\mathrm{I}-}$ and $\mathrm{B} 6^{\mathrm{II}-}$ tracheas had occlusions of $53.0 \%$ $\pm 16.3 \%$ and $52.2 \% \pm 15.9 \%$, respectively $(P=.20, .19)$. In chimeric B6 tracheas, minimal occlusion was seen at day 14 and remained $33.6 \% \pm 16.2 \%$ $(P=.039)$ at day 28. Subtle epithelial changes and minimal infiltrates were seen.

Conclusions: Obliterative airway disease appears to involve donor-type antigen-presenting cells and develops in the absence of either MHC class I or II antigens. These findings suggest that either $\mathrm{CD}^{+}$or $\mathrm{CD}^{+}$direct allorecognition is important in the development of obliterative airway disease.

(J Thorac Cardiovasc Surg 2000;120:1070-7)
From the Department of Surgery, ${ }^{a}$ Division of Cardiothoracic Surgery, and the Department of Pathology and Laboratory Medicine, ${ }^{\mathrm{b}}$ Hospital of the University of Pennsylvania, Philadelphia, Pa.

Supported in part by the American Surgical Association, the Charles E. Culpeper Foundation, and The American Association for Thoracic Surgery, Dwight Harken Research Scholarship.

Read at the Eightieth Annual Meeting of The American Association for Thoracic Surgery, Toronto, Ontario, Canada, April 30-May 3, 2000.

Received for publication May 4, 2000; revisions requested June 20, 2000; revisions received July 21, 2000; accepted for publication Aug 3, 2000.

Address for reprints: Bruce R. Rosengard, MD, Division of Cardiothoracic Surgery, Department of Surgery, Hospital of the University of Pennsylvania, 3400 Spruce St, Silverstein 6th Floor, Philadelphia, PA 19104

(E-mail: brosenga@mail.med.upenn.edu).

Copyright (†) 2000 by The American Association for Thoracic Surgery

0022-5223/2000 $\$ 12.00+0 \quad \mathbf{1 2 / 6 / 1 1 0 6 7 4}$

doi: $10.1067 / \mathrm{mtc} .2000 .110674$ ung transplantation has become a widely accepted Lform of therapy for end-stage lung disease, with approximately 10,000 lung transplantation procedures performed throughout the world. ${ }^{1}$ As a result of improvements in surgical techniques and immunosuppression, the short-term survival after lung transplantation has improved dramatically, with 1-year survival currently approaching $70 \% .^{1}$ However, the current 5year survival is $43.3 \%$ with a median survival of 3.7 years. ${ }^{1}$ Long-term survival in lung transplantation has been limited primarily by the development of obliterative bronchiolitis (OB). The incidence of OB is $28 \%$ at 1 year after transplantation and reaches $60 \%$ to $80 \%$ after 5 to 10 years. ${ }^{2,3}$ OB is characterized by a fibroproliferative process that predominately involves the smaller airways. Histologic features include luminal obliteration of the airway accompanied by lymphocytic infiltrates and epithelial desquamation and necrosis. ${ }^{4}$ 
The pathogenesis of OB remains poorly understood, but the histologic characteristics and the absence of the process in experimental animals rendered tolerant have led many to speculate that $\mathrm{OB}$ is the primary manifestation of chronic rejection in lung transplantation. Clinical observations support the notion that $\mathrm{OB}$ results from alloimmune-mediated mechanisms. Acute rejection has been demonstrated to be a major risk factor for the subsequent development of OB. ${ }^{5-7} \mathrm{Up}$-regulation of major histocompatibility complex (MHC) class I and class II antigens on bronchial epithelium and infiltration of donor-specific lymphocyte subsets have been found in grafts with OB. ${ }^{8-10}$ The presence of anti-HLA class I antibodies in lung transplant recipients is associated with $\mathrm{OB}$, and recently anti-HLA class I antibodies have been shown to lead to protein phosphorylation and proliferation of cultured lung epithelium. ${ }^{11}$ Furthermore, increased immunosuppression has been shown to stabilize lung function in some patients. ${ }^{12,13}$

Bone marrow-derived antigen-presenting cells (APCs) play a central role in triggering recipient $\mathrm{T}$ cells. ${ }^{14}$ However, little is known about how allorecognition pathways affect the development of OB. Responses to allogeneic tissue are unique in that both donor and recipient APCs are capable of triggering rejection, termed direct and indirect allorecognition, respectively. Both recipient $\mathrm{CD} 4^{+}$and $\mathrm{CD} 8^{+} \mathrm{T}$ cells can be triggered directly by donor APCs, whereas recipient APCs stimulate only $\mathrm{CD}^{+}{ }^{+} \mathrm{T}$ cells by presenting allopeptides in the context of self MHC class II molecules. Understanding the role of allorecognition pathways in OB may lead to the development of rational strategies to prevent this malady.

The goal of our study was to investigate whether direct allorecognition triggered by donor APCs is important in the development of $\mathrm{OB}$, and, if so, which pathways are involved. We used a heterotopic mouse tracheal transplant model to study the development of obliterative airway disease, which resembles OB histologically. ${ }^{15}$ To eliminate the direct allorecognition pathway, we created chimeric B6 tracheas bearing recipient $\mathrm{C} 3 \mathrm{H}$ APCs, denoted hereafter as $\mathrm{B} 6(\mathrm{C} 3 \mathrm{H})$, using allogeneic bone marrow transplantation (BMT), ${ }^{16,17}$ and transplanted them into $\mathrm{C} 3 \mathrm{H}$ recipients. Furthermore, tracheas from B6 MHC class I knockout $\left(\mathrm{B}^{\mathrm{I}-}\right)$ mice and $\mathrm{B} 6 \mathrm{MHC}$ class II knockout $\left(\mathrm{B}^{\mathrm{II}-}\right)$ mice were transplanted to examine the contributions of $\mathrm{CD}^{+}$and $\mathrm{CD} 8^{+}$direct allorecognition pathways. Naive or autologously reconstituted $\mathrm{B} 6$ and $\mathrm{C} 3 \mathrm{H}$ tracheas were transplanted into $\mathrm{C} 3 \mathrm{H}$ recipients as our positive and negative controls, respectively. The autologously reconstituted B6 tracheas, denoted hereafter as B6(B6), behaved similarly to the naive B6 and served as controls for the BMT tracheas. We report here that donor bone marrow-derived APCs are important in the development of obliterative airway disease and that donor APC expression of either MHC class I or class II antigen is sufficient to trigger the development of obliterative airway disease.

\section{Materials and methods}

Experimental animals. All laboratory animals received humane care in compliance with the "Guide for the Care and Use of Laboratory Animals" prepared by the Institute of Laboratory Animal Resources and published by the National Institutes of Health (NIH Publication No. 86-23, revised 1985). All animals were housed in conventional cages with filter lids and provided access to food and water. Donor C57BL/6 (B6, H-2 $2^{\mathrm{b}}$ ), MHC class I knockout ( $\$ 2 \mathrm{~m}$ deficient) on the C57/BL6 background (B6 $\left.{ }^{\mathrm{I}}\right)$ mice, and MHC class II knockout (I-A $\mathrm{A}^{\mathrm{b}}$ B-chain deficient) on the C57/BL6 background $\left(\mathrm{B} 6^{\mathrm{II}-}\right)$ mice, 5 to 7 weeks of age, were purchased from Taconic Laboratories (Germantown, NY). C3H/HeJ (H$\left.2^{\mathrm{k}}\right)$ mice, 5 to 7 weeks of age, were purchased from Jackson Laboratory (Bar Harbor, Maine).

BMT. Chimeric tracheas were created by BMT as previously described. ${ }^{16,17}$ In brief, BMT recipient mice were lethally irradiated $(10 \mathrm{~Gy})$ and then reconstituted with $1 \times 10^{7}$ unfractionated bone marrow cells from a donor, injected in 1 $\mathrm{mL}$ of medium via the tail vein. The BMT recipients were treated with 2 weeks of oral antibiotics and allowed to engraft for 90 days before their use as trachea donors.

Heterotopic tracheal transplantation. By means of a technique first described by Hertz and associates, ${ }^{15} 1$ to 4 tracheal segments were transplanted subcutaneously into the dorsa of $\mathrm{C} 3 \mathrm{H}$ recipients in experimental groups as shown in Table I. Recipients with multiple tracheal segments contained grafts from the same genotypes. No immunosuppression was administered to any group. Donor mice were anesthetized with enflurane, and their skin was prepared with ethyl alcohol. In each case, the thoracic cavity was entered through a midline incision, and the animal was exsanguinated. The thymus and the heart were removed, so that the trachea and the main bronchi were exposed. With sharp dissection, the trachea from the larynx to the carina was removed and placed in cold Dulbecco's phosphate-buffered saline solution (Gibco, Grand Island, NY).

Recipient mice were similarly anesthetized, and their dorsa were shaved and prepared with ethyl alcohol. A small skin incision was made on their dorsa and the tracheal segments were implanted subcutaneously. The incision was closed with 4-0 Vicryl sutures (Ethicon, Inc, Somerville, $\mathrm{NJ}$ ). The time interval between harvest and implantation was less than 10 minutes.

Confirmation of chimerism with flow cytometry. To confirm the status of bone marrow chimeras, we analyzed flow cytometry as previously described by our laboratory. ${ }^{16,17}$ We have previously shown that the degree of APC replacement in the spleen consequent to BMT mirrors the 


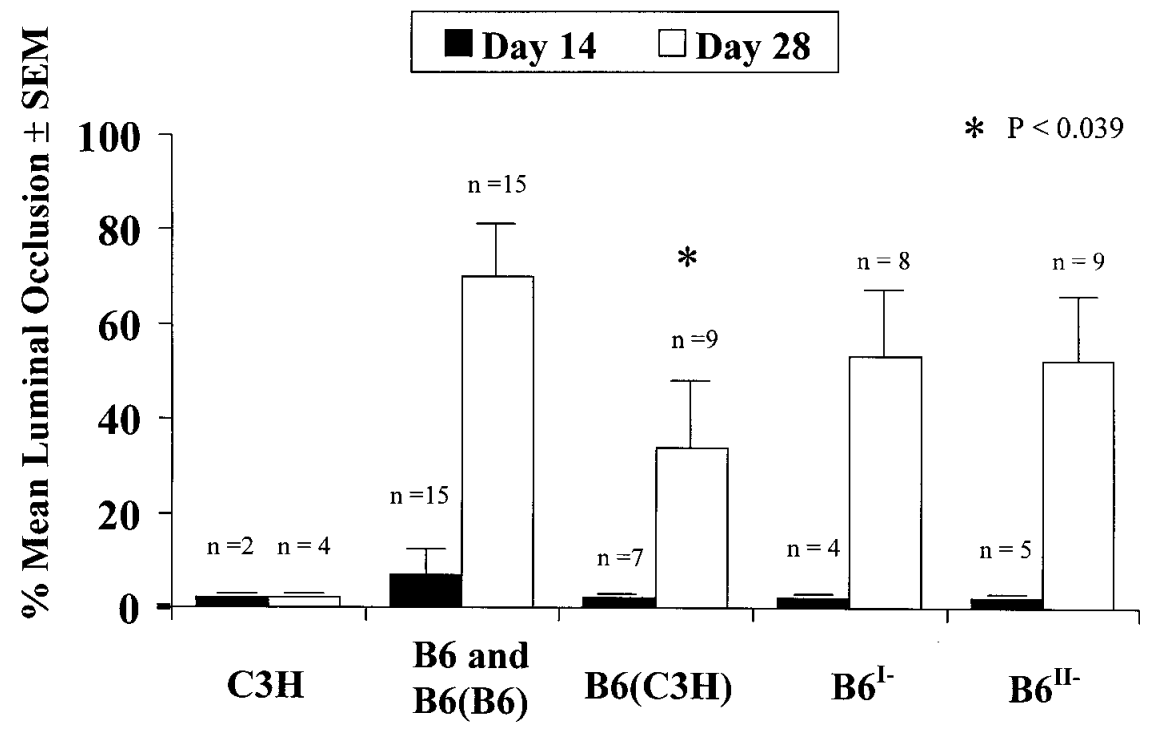

Fig 1. The mean luminal occlusion in $\mathrm{C} 3 \mathrm{H}, \mathrm{B} 6$ and $\mathrm{B} 6(\mathrm{~B} 6)$, and $\mathrm{B} 6(\mathrm{C} 3 \mathrm{H}), \mathrm{B} 6^{\mathrm{I}-}$, and $\mathrm{B} 6^{\mathrm{II}-}$ tracheas on days 14 and 28 ( $\mathrm{n}=$ number of recipients). All tracheas were transplanted into $\mathrm{C} 3 \mathrm{H}$ recipients. On day 28 , a significant decrease in the degree of luminal occlusion was noted in the $\mathrm{B} 6(\mathrm{C} 3 \mathrm{H})$ group compared with the B6 and B6(B6) group $(P=.039)$.

Table I. Experimental groups

\begin{tabular}{lccc}
\hline Tracheal donor & Parenchyma & APCs & Recipient \\
\hline $\mathrm{C} 3 \mathrm{H}$ & $\mathrm{C} 3 \mathrm{H}$ & $\mathrm{C} 3 \mathrm{H}$ & $\mathrm{C} 3 \mathrm{H}$ \\
$\mathrm{B} 6 / \mathrm{B} 6(\mathrm{~B} 6)$ & $\mathrm{B} 6$ & $\mathrm{~B} 6$ & $\mathrm{C} 3 \mathrm{H}$ \\
$\mathrm{B} 6(\mathrm{C} 3 \mathrm{H})$ & $\mathrm{B} 6$ & $\mathrm{C} 3 \mathrm{H}$ & $\mathrm{C} 3 \mathrm{H}$ \\
$\mathrm{B}^{\mathrm{I}} \mathrm{-}$ & $\mathrm{B} 66^{\mathrm{I}}$ & $\mathrm{B} 6^{\mathrm{I}}$ & $\mathrm{C} 3 \mathrm{H}$ \\
B6 $^{\mathrm{II}-}$ & $\mathrm{B}^{\mathrm{II}-}$ & $\mathrm{B}^{\mathrm{II}-}$ & $\mathrm{C} 3 \mathrm{H}$ \\
\hline
\end{tabular}

$B 6(B 6)$, B6 autologously reconstituted with B6 bone marrow-derived APCs; $B 6(C 3 H)$, B6 reconstituted with $\mathrm{C} 3 \mathrm{H}$ bone marrow-derived APCs; $B 6^{I-}$, MHC class I antigen knockout B6; $B 6^{H-}$, MHC class II antigen knockout B6.

degree of APC replacement in other organs. This method has been further validated by reverse transcriptase-polymerase chain reaction and the mixed lymphocyte reaction. Spleens of donor chimeric animals were harvested at the time of transplantation. The spleens were mechanically disrupted and filtered over a $70-\mu \mathrm{m}$ sterile nylon mesh. The spleen cells were treated with ACK buffer (BioWhittaker, Walkersville, Md), washed with cold phosphate-buffered saline solution, and counted. All antibodies were obtained from Pharmingen (San Diego, Calif). Fluorescein isothiocyanate-conjugated mouse anti-mouse $\mathrm{I}-\mathrm{A}^{\mathrm{b}}$ (clone 25-9-17, immunoglobulin G2a) and fluorescein isothiocyanate-conjugated mouse anti-mouse $\mathrm{I}^{-\mathrm{A}^{\mathrm{k}}}$ (11-5.2, immunoglobulin $\mathrm{G} 2 \mathrm{~b}$ ) were used to label spleen cells from chimeras for flow cytometry analysis. Flow cytometry analysis was performed on a FACScan flow cytometer equipped with CELLQuest software (Becton Dickinson, San Jose, Calif).

Graft removal and data analysis. Tracheal grafts were removed 14 and 28 days after transplantation. In recipients with multiple grafts, the tracheal segments were removed at the same time points. Specimens were fixed in $10 \%$ formalin and submitted for staining with hematoxylin and eosin. All tracheal specimens were evaluated for their degree of luminal occlusion, the morphology of the respiratory epithelium, and the presence of intraepithelial and peritracheal cellular infiltrates by a pathologist blinded to the protocol. For recipients with multiple tracheal segments, the mean luminal occlusion of the grafts was reported. The Student $t$ test was used to compare the mean luminal occlusion in all groups versus that of the naive and autologously reconstituted B6 allograft group.

\section{Results}

Confirmation of chimerism. Spleens from all BMT recipients were analyzed to confirm complete APC replacement at the time of tracheal harvest for transplantation (data not shown). Tracheas from donors having incomplete APC replacement were not included in this study.

Luminal occlusion. Luminal occlusion was determined by microscopy and expressed as a percentage of the cross-sectional area of the tracheal lumen. The mean luminal occlusion of the groups was compared as shown in Fig 1. No statistically significant difference existed between the mean occlusion of the naive B6 and the autologously reconstituted B6(B6) grafts at day $28(P=.43)$. Hence, all B6 and B6(B6) grafts were placed into the allograft group. At days 14 and 28, none of the isografts showed evidence of luminal occlusion. In the allograft group at day 14 , the luminal occlusion was $5.7 \% \pm 5.4 \%$ (mean \pm standard error of the mean). 

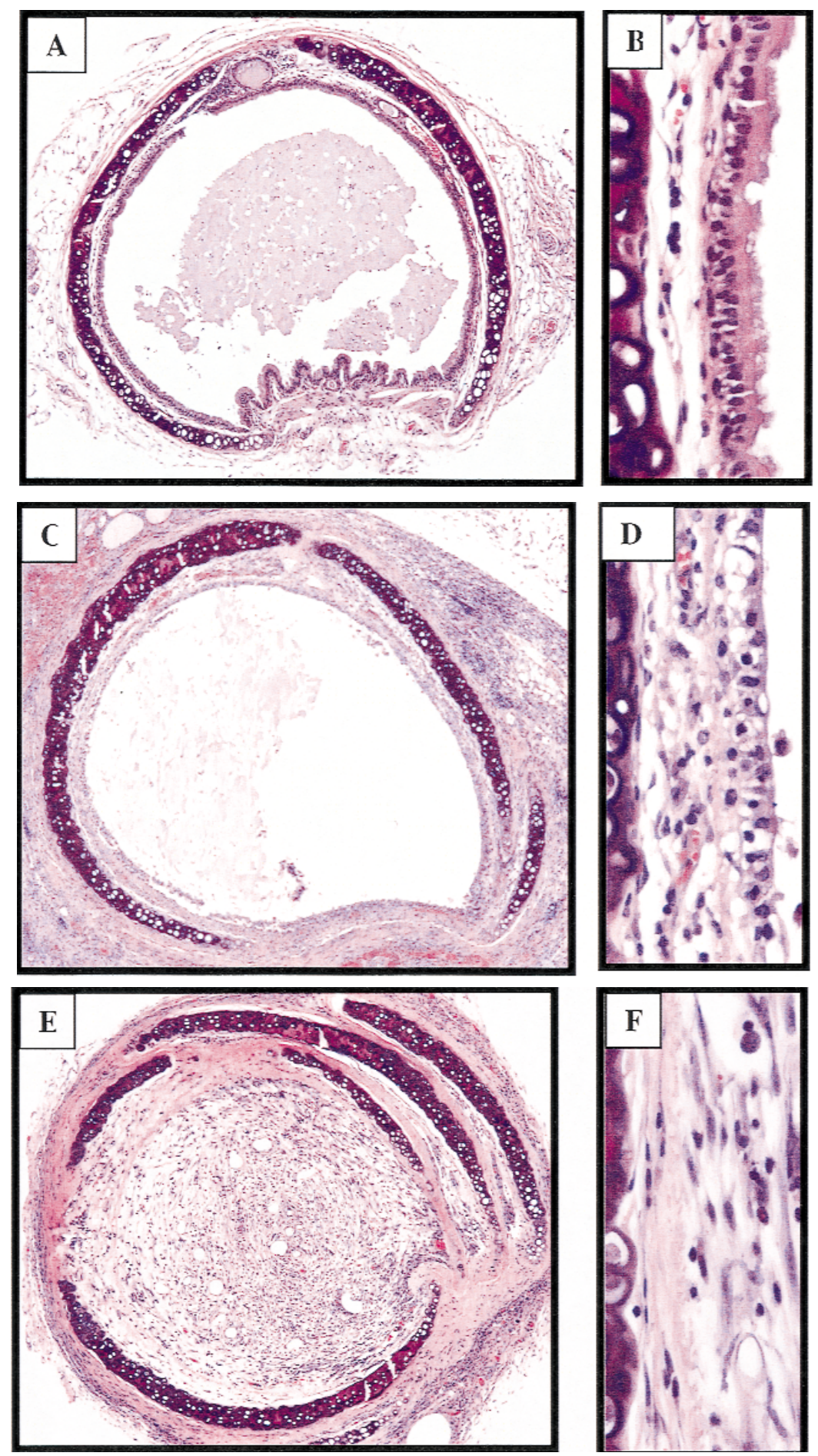

Fig 2. Photomicrographs of tracheal specimens in cross section stained with hematoxylin and eosin. A and $\mathbf{B}$, Tracheal sections representative of $\mathrm{C} 3 \mathrm{H}$ isografts. A, Isografts are patent with minimal peritracheal inflammation at day $28(40 \times)$. B, Normal ciliated respiratory epithelium is seen lining the lumen, and intraepithelial lymphocytes are absent (400×). C to F, Tracheal sections representative of B6, B6(B6), B6 ${ }^{\mathrm{I}}$, and B6 ${ }^{\mathrm{II}-}$ allografts. At day 14, (C) the lumina are patent with moderate peritracheal inflammation $(40 \times)$, and $(\mathbf{D})$ the respiratory epithelium is flattened, nonciliated, and infiltrated with inflammatory cells $(400 \times)$. At day 28, (E) the allografts are completely occluded and have minimal peritracheal inflammation $(40 \times)$; $\mathbf{F}$, the lumina are filled with loose connective tissue containing scant inflammatory cells, which have replaced the epithelium and submucosal tissue (400×). 

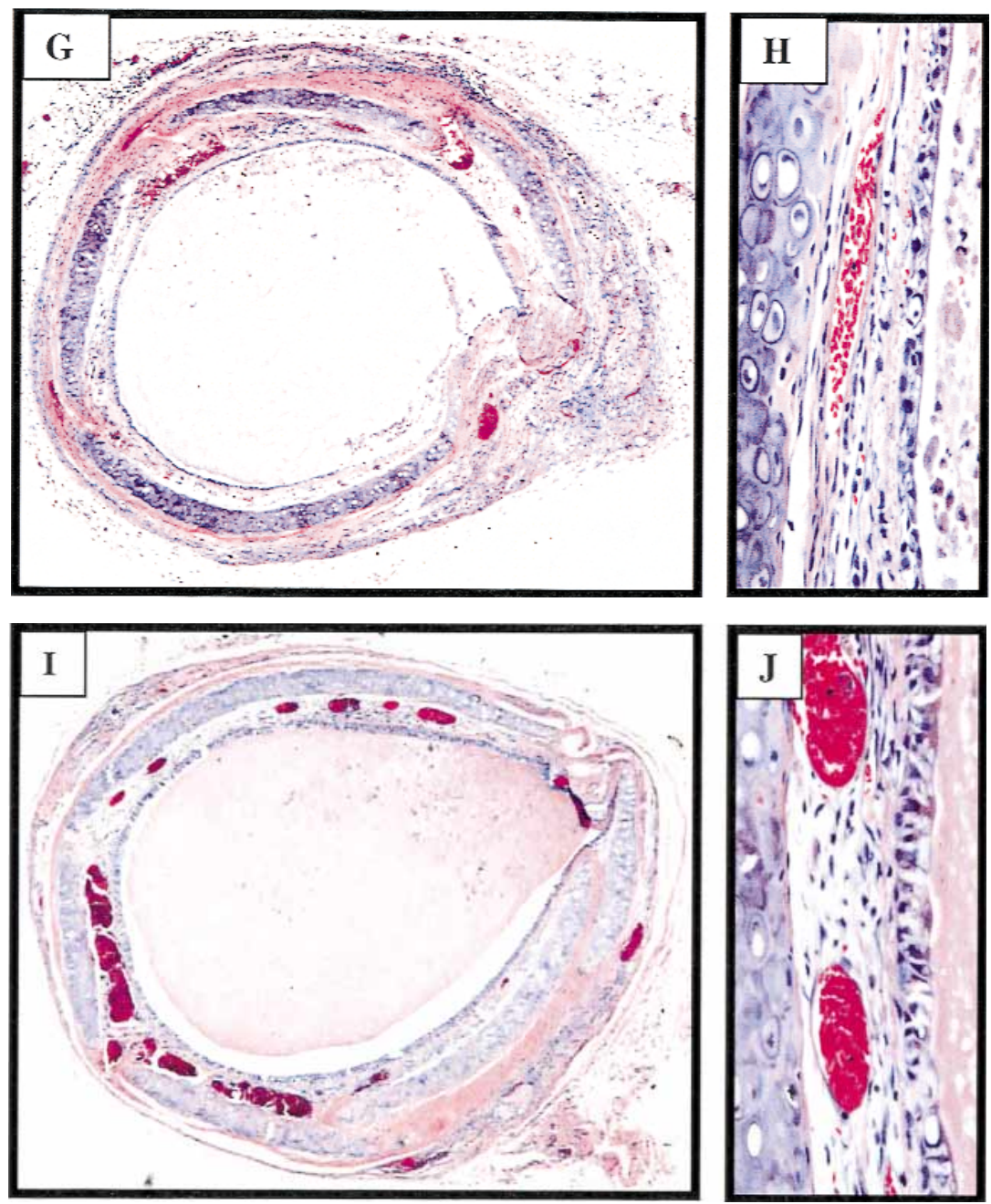

Fig 2. Cont'd. G to J, Tracheal sections representative of B6(C3H) grafts. At day 14, (G) the lumina are patent $(40 \times)$ and $(\mathbf{H})$ the respiratory epithelium is similar to that seen in allografts; the epithelium is flattened, nonciliated, and infiltrated with inflammatory cells (400×). At day 28, (I) the lumina are patent with minimal peritracheal inflammation $(40 \times)$ and $(\mathbf{J})$ the epithelium remains intact, although flattened and nonciliated with minimal cellular infiltrates $(400 \times)$.

Specimens from 14 of 15 recipients demonstrated minimal occlusion (ranging from $0 \%$ to $5 \%$ ), and 1 of 15 demonstrated marked occlusion $(80 \%)$. At day 28 , the luminal occlusion was $69.5 \% \pm 11.6 \%$. Specimens from 10 of 15 recipients demonstrated marked occlusion (ranging from $90 \%$ to $100 \%$ ), and 5 of 15 demonstrated minimal to moderate occlusion (ranging from $0 \%$ to $50 \%$ ). In the $\mathrm{B}^{\mathrm{I}-}$ and $\mathrm{B} 6^{\mathrm{II}-}$ groups, tracheas showed minimal occlusion at day 14 (ranging from $0 \%$ to $5 \%$ ). At day 28 in the $\mathrm{B}^{\mathrm{I}}{ }^{-}$group, the luminal occlusion was $53.0 \% \pm 16.3 \%$. Specimens from 4 of 8 recipients demonstrated marked occlusion (ranging from $70 \%$ to $100 \%$ ), and 4 of 8 had moderate occlusion (ranging from $0 \%$ to $55 \%$ ). Similarly, the luminal occlusion in the $\mathrm{B} 6{ }^{\mathrm{II}-}$ group at day 28 was $52.2 \% \pm$ $15.9 \%$. Specimens from 4 of 9 recipients had marked occlusion (100\%), and 5 of 9 demonstrated moderate occlusion (ranging from $0 \%$ to $50 \%$ ). Tracheas from the $\mathrm{B} 6^{\mathrm{I}-}$ and $\mathrm{B} 6^{\mathrm{II}-}$ groups did not demonstrate significant decreases in luminal occlusion at day 28 when compared with the allograft group $(P=.20, .19$, respectively). In the $\mathrm{B} 6(\mathrm{C} 3 \mathrm{H})$ group at day 14 , the luminal occlusion was $0.7 \% \pm 0.7 \%$. Specimens from all 7 recipients demonstrated minimal occlusion (ranging from $0 \%$ to $5 \%$ ). At day 28, the luminal occlusion was $33.6 \% \pm 16.2 \%$. Specimens from 6 of 9 recipients 
demonstrated minimal occlusion (ranging from $0 \%$ to $5 \%$ ), and 3 of 9 demonstrated marked occlusion (ranging from $90 \%$ to $100 \%)$. B6 $(\mathrm{C} 3 \mathrm{H})$ tracheas demonstrated a significant decrease in luminal occlusion when compared with the allograft group at day $28(P=$ .039).

Epithelial morphology and cellular infiltrates. The respiratory epithelial morphology, peritracheal inflammation, and intraepithelial cellular infiltrates were characterized and compared between the groups. In the isograft group at both days 14 and 28, the respiratory epithelial lining remained intact and was predominately ciliated columnar epithelial cells. Minimal to mild peritracheal inflammation and intraepithelial cellular infiltrates were seen (Fig 2, $A$ and $B$ ). In the B6 and B6(B6) group at day 14, the respiratory epithelium remained present, but the cells were predominately flattened and nonciliated. Moderate to severe peritracheal inflammation and intraepithelial cellular infiltrates were present (Fig 2, $C$ and $D$ ). By day 28, completely occluded allografts showed no evidence of the respiratory epithelium. The amount of peritracheal inflammation and cellular infiltrates had diminished when compared with the observations on day 14 (Fig 2, $E$ and $F$ ). In the $\mathrm{B} 6(\mathrm{C} 3 \mathrm{H})$ group at day 14 , flattened or atypical, nonciliated epithelium lined the lumina, much like the allograft group. Mild to moderate amounts of cellular infiltrates were seen in the epithelium and the peritracheal tissue. Compared with the allograft group, the inflammatory events appeared to be slightly diminished (Fig 2, $G$ and $H$ ). Through day 28, flattened or nonciliated epithelium persisted in chimeric tracheas with nonoccluded lumina. Even though peritracheal and intraepithelial infiltrates persisted, they also appeared to be diminished when compared with day 14 (Fig 2, I and $J$ ). In both the $\mathrm{B}^{\mathrm{I}-}$ and the $\mathrm{B}^{\mathrm{II}-}$ groups, the epithelial changes and the severity of cellular infiltrates were similar to those of the naive and autologously reconstituted B6 allografts at days 14 and 28.

\section{Discussion}

OB is a devastating long-term complication of lung transplantation, affecting at least $50 \%$ of lung transplant recipients surviving beyond 3 years. ${ }^{18}$ The progressive decrease in pulmonary function associated with $\mathrm{OB}$ has a profound impact on the quality of life of these patients, and OB remains the primary cause of late death after lung transplantation. Currently, therapeutic options are limited to retransplantation and intensification of immunosuppression. However, these strategies have limited success. A better understanding of the pathogenesis of $\mathrm{OB}$ is necessary if rational strategies are to be developed for its prevention and treatment.

Evidence from human studies has suggested that $\mathrm{OB}$ is an alloimmune-mediated process against the bronchial epithelium. Increased immunosuppression has been shown to stop the progression of $\mathrm{OB}$ in some patients. ${ }^{12,13}$ Presentation of MHC class I and class II antigens on the bronchial epithelium has been demonstrated to be a possible mechanism in the development of OB. ${ }^{8-11,19-21}$ Professional APCs such as dendritic cells are associated with bronchial tissues of patients with $\mathrm{OB}$ and have been postulated to be involved in the development of OB. ${ }^{22}$

Animal models have also been described to study the relationship between the immune responses and the development of OB. Orthotopic allogeneic lung transplants in rats and pigs develop the lesions of $\mathrm{OB} .^{23,24}$ However, these models require substantial surgical expertise, and $\mathrm{OB}$ does not consistently develop in the small animal models. The mouse heterotopic tracheal allograft model of obliterative airway disease was first described by Hertz and associates ${ }^{15}$ in 1993. This model reproduces some of the histopathologic characteristics of $\mathrm{OB}$ over a period of 3 to 4 weeks.

Data from animal studies support the notion that $\mathrm{OB}$ is a result of histoincompatibility. Expression of $\mathrm{MHC}$ class II has been shown to be increased on the bronchial epithelium in rat lung allografts with $\mathrm{OB} .^{23,24}$ Furthermore, Pham and colleagues ${ }^{25}$ demonstrated that induction of tolerance by means of mixed hematopoietic chimerism prevents $\mathrm{OB}$ in rat orthotopic lung allografts. Similarly, Kelly, Hertz, and Mueller ${ }^{26}$ demonstrated that alloimmune responses directed against class I antigens on bronchial epithelium may be responsible for the development of obliterative airway disease. Moreover, several groups have shown that the use of immunosuppression can prevent the development of obliterative airway disease. ${ }^{27,28}$

The present study provides further evidence that $\mathrm{OB}$ is an immune-mediated process. Consistent with the findings in human and animal studies, our data suggest that professional APCs may play a central role in the development of obliterative airway disease. ${ }^{22-24} \mathrm{By}$ replacing donor-type APCs with recipient-type APCs, we eliminated direct allorecognition of allopeptides presented by donor-type APCs and prevented the development of obliterative airway disease. Similar to the B6 and B6(B6) allografts, the $\mathrm{B} 6(\mathrm{C} 3 \mathrm{H})$ specimens exhibited evidence of an early inflammatory event at day 14 that subsided by day 28 . However, in contrast to the B6 and B6(B6) allografts, luminal occlusion of the $\mathrm{B} 6(\mathrm{C} 3 \mathrm{H})$ specimens remained minimal at day 28 , and 
although flattened and nonciliated, the respiratory epithelium remained intact. Interestingly, immunosuppressive regimens including cyclosporine (INN: ciclosporin) and sirolimus have been shown to inhibit the development of obliterative airway disease and have resulted in similar epithelial changes with minimal luminal occlusion. ${ }^{27,28}$ The pathogenesis of $\mathrm{OB}$ is thought to involve an early epithelial injury followed by aberrant epithelial regeneration related to the alloimmune response. Our findings are consistent with this paradigm since $\mathrm{B} 6(\mathrm{C} 3 \mathrm{H})$ tracheas demonstrate early epithelial injury but undergo a near-normal process of repair, presumably as a consequence of reduced immunogenicity of the grafts. Tracheas from autologously reconstituted B6 mice confirmed that the lack of obliterative airway disease was not due to the effects of irradiation or BMT.

Our findings involving the $\mathrm{B} 6^{\mathrm{I}-}$ and the $\mathrm{B} 6^{\mathrm{II}-}$ tracheas have further demonstrated that "professional" APCs expressing either MHC class I or class II antigens are sufficient to trigger the development of obliterative airway disease. Previous human and animal studies have suggested that MHC class I and class II antigens on bronchial epithelium (a "nonprofessional" APC) are sufficient to drive the development of OB. ${ }^{8-11,19-21,23,26}$ In contrast, our data suggest that direct presentation by the bronchial epithelium or indirect presentation of donor MHC class I and class II antigens by recipient APCs is not sufficient to drive the development of obliterative airway disease.

Increasing evidence suggests that chronic rejection is mediated by persistent indirect presentation of allopeptides. Vella and coworkers ${ }^{29}$ demonstrated that lymphocytes from renal transplant recipients with chronic rejection had a significant increase in proliferation to HLA peptides when compared with controls. Ciubotariu and associates ${ }^{30}$ showed that the incidence of cardiac allograft vasculopathy was significantly higher in patients whose lymphocytes displayed persistent allopeptide reactivity. Last, our laboratory has previously shown that chimeric rat cardiac allografts bearing recipient-type APCs in a low responder strain combination survive long term but have severe allograft vasculopathy. ${ }^{17}$

In our experiments, obliterative airway disease does not develop in chimeric tracheas in the absence of donor-type APCs, despite the presence of bronchial epithelial MHC class I and class II antigens. Therefore, we believe that obliterative airway disease is a model of acute rejection, rather than one of chronic rejection. It has been postulated that the vigor of acute allograft rejection is due to the "unnatural" stimulation of recipient $\mathrm{T}$ cells by donor-type APCs (direct allorecognition). The ability of graft-resident bone marrow-derived APCs to induce allosensitization was first demonstrated by Steinmuller. ${ }^{31}$ Subsequently, Lechler and Batchelor ${ }^{14}$ showed that depletion of donor APCs led to prolonged allograft survival and that perioperative adoptive transfer of donor-type APCs restored acute rejection. Recently, our laboratory has reported that donor APC replacement in the same mouse strain combination alters the tempo and pathogenesis of cardiac allograft rejection. ${ }^{17}$ One caveat is that obliterative airway disease might eventually develop in chimeric tracheas if subsequent time points were assessed. However, the inability in our laboratory to harvest intact chimeric specimens (previously irradiated) with consistently reliable architecture beyond 28 days makes it difficult to address this possibility experimentally.

Even though the fibroproliferative process in obliterative airway disease resembles that found in $\mathrm{OB}$ histologically, the murine heterotopic tracheal transplant model has several limitations. The lesions of $\mathrm{OB}$ are primarily found in the terminal airways in human beings, whereas the obliterative airway disease lesion occurs more proximally in the airway. In addition, the heterotopic tracheal transplant model depends on neovascularization, in contrast to immediately revascularized orthotopic lung transplant models. Although this system has been used as a model of $\mathrm{OB}$, the failure of therapies that effectively prevent obliterative airway disease to make an impact on clinical $\mathrm{OB}$ further suggests that this model should be used in studies of acute rejection.

In summary, our study demonstrates that donor-type APCs are important in the development of obliterative airway disease in a heterotopic tracheal transplant model. Furthermore, donor APC expression of either MHC class I or class II antigens is sufficient to trigger the development of obliterative airway disease. In terms of clinical applicability, the concept of APC depletion to reduce immunogenicity of allografts has already led to clinical trials with anti-CD45 used in renal transplantation..$^{32,33}$ On the basis of these studies, we would speculate that APC depletion might reduce the incidence and severity of acute lung rejection.

\section{REFERENCES}

1. The Registry of the International Society for Heart and Lung Transplantation: Seventeenth Annual Report, 2000.

2. Cooper JD, Patterson GA, Trulock EP. Results of single and bilateral lung transplantation in 131 consecutive recipients: Washington University Lung Transplant Group. J Thorac Cardiovasc Surg 1994;107:460-70.

3. Valentine VG, Robbins RC, Berry GJ, et al. Actuarial survival of 
heart-lung and bilateral sequential lung transplant recipients with obliterative bronchiolitis. J Heart Lung Transplant 1996;15:371-83.

4. Yousem SA, Berry GJ, Cagle PT, et al. Revision of the 1990 working formulation for the standardization of nomenclature in the diagnosis of heart and lung rejection: Lung Rejection Study Group. J Heart Lung Transplant 1996;15:1-15.

5. Bando K, Paradis IL, Similo S, et al. Obliterative bronchiolitis after lung and heart-lung transplantation: an analysis of risk factors and management. J Thorac Cardiovasc Surg 1995;110:4-14.

6. Reichenspurner H, Girgis RE, Robbins RC, et al. Stanford experience with obliterative bronchiolitis after lung and heart-lung transplantation. Ann Thorac Surg 1996;62:1467-73.

7. Heng D, Sharples LD, McNeil K, Stewart S, Wreghitt T, Wallwork J. Bronchiolitis obliterans syndrome: incidence, natural history, prognosis, and risk factors. J Heart Lung Transplant 1998;17:1255-63.

8. Hasegawa S, Ockner DM, Ritter JH, et al. Expression of class II major histocompatibility complex antigens (HLA-DR) and lymphocyte subset immunotyping in chronic pulmonary transplant rejection. Arch Pathol Lab Med 1995;119:432-9.

9. Reinsmoen NL, Bolman RM, Savik K, Butters K, Hertz M. Differentiation of class I and class II directed donor specific alloreactivity in bronchoalveolar lavage lymphocytes from lung transplant recipients. Transplantation 1992;53:181-9.

10. Taylor PM, Rose ML, Yacoub MH. Expression of MHC antigens in normal human lungs and transplanted lungs with obliterative bronchiolitis. Transplantation 1989;48:506-10.

11. Reznik SI, Jaramillo A, Zhang L, Patterson GA, Cooper JD, Mohanakumar T. Anti-HLA antibody binding to HLA class I molecules induces proliferation of airway epithelial cells: a potential mechanism for bronchiolitis obliterans syndrome. J Thorac Cardiovasc Surg 2000;119:39-45.

12. Kesten S, Rajagopalan N, Maurer J. Cytolytic therapy for the treatment of bronchiolitis obliterans syndrome following lung transplantation. Transplantation 1996;61:427-30.

13. Dusmet M, Maurer J, Winton J, Kesten S. Methotrexate can halt the progression of bronchiolitis obliterans syndrome in lung transplant recipients. J Heart Lung Transplant 1996;15:948-54.

14. Lechler RI, Batchelor JR. Restoration of immunogenicity to passenger cell depleted kidney allografts by the addition of donor strain dendritic cells. J Exp Med 1982;155:31-41.

15. Hertz MI, Jessurun J, King MB, Savik SK, Murray JJ. Reproduction of the obliterative bronchiolitis lesion after heterotopic transplantation of mouse airways. Am J Pathol 1993;142:1945-51.

16. Eiref SD, Zhang W, Popma SH, Shah LJ, Moore JS, Rosengard BR. Creation of chimeric hearts: a tool for testing the "passenger leukocyte" hypothesis. Ann Thorac Surg 1997;64:628-33.

17. Krasinskas AM, Eiref SD, McLean AD, Kreisel D, Gelman AE, Popma SH, et al. Replacement of graft-resident donor-type antigen presenting cells alters the tempo and pathogenesis of murine cardiac allograft rejection. Transplantation 2000;70:514-21.

18. Sundaresan S, Trulock EP, Mohanakumar T, Cooper JD, Patterson
GA. Prevalence and outcome of bronchiolitis obliterans syndrome after lung transplantation. Ann Thorac Surg 1995;60:1341-6.

19. SivaSai KS, Smith MA, Poindexter NJ, et al. Indirect recognition of donor HLA class I peptides in lung transplant recipients with bronchiolitis obliterans syndrome. Transplantation 1999;67:1094-8.

20. Nakajima J, Poindexter NJ, Hillemeyer PB, et al. Cytotoxic T lymphocytes directed against donor HLA class I antigens on airway epithelial cells are present in bronchoalveolar lavage fluid from lung transplant recipients during acute rejection. J Thorac Cardiovasc Surg 1999;117:565-71.

21. Rizzo M, Sundaresan S, Lynch J, et al. Increased concentration of soluble human leukocyte antigen class I levels in the bronchoalveolar lavage of human pulmonary allografts. J Heart Lung Transplant 1997; 16:1135-40.

22. Yousem SA, Ray L, Paradis IL, Dauber JA, Griffith BP. Potential role of dendritic cells in bronchiolitis obliterans in heart-lung transplantation. Ann Thorac Surg 1990;49:424-8.

23. Uyama T, Winter JB, Groen G, Wildevuur CR, Monden Y, Prop J. Late airway changes caused by chronic rejection in rat lung allografts. Transplantation 1992;54:809-12.

24. Al-Dossari GA, Kshettry VR, Jessurun J, Bolman RM III. Experimental large-animal model of obliterative bronchiolitis after lung transplantation. Ann Thorac Surg 1994;58:34-9.

25. Pham SM, Mitruka SN, Youm W, et al. Mixed hematopoietic chimerism induces donor-specific tolerance for lung allografts in rodents. Am J Respir Crit Care Med 1999;159:199-205.

26. Kelly KE, Hertz MI, Mueller DL. T-cell and major histocompatibility complex requirements for obliterative airway disease in heterotopically transplanted murine tracheas. Transplantation 1998;66:764-71

27. King MB, Jessurun J, Savik SK, Murray JJ, Hertz MI. Cyclosporine reduces development of obliterative bronchiolitis in a murine heterotopic airway model. Transplantation 1997;63:528-32.

28. Fahrni JA, Berry GJ, Morris RE, Rosen GD. Rapamycin inhibits development of obliterative airway disease in a murine heterotopic airway transplant model. Transplantation 1997;63:533-7.

29. Vella JP, Spadafora-Ferreira M, Murphy B, et al. Indirect allorecognition of major histocompatibility complex allopeptides in human renal transplant recipients with chronic graft dysfunction. Transplantation 1997;64:795-800.

30. Ciubotariu R, Liu Z, Colovai AI, et al. Persistent allopeptide reactivity and epitope spreading in chronic rejection of organ allografts. J Clin Invest 1998;101:398-405.

31. Steinmuller D. Immunization with skin isografts taken from tolerant mice. Science 1967;158:127-9.

32. Goldberg LC, Bradley JA, Connolly J, et al. Anti-CD45 monoclonal antibody perfusion of human renal allografts prior to transplantation: a safety and immunohistological study. CD45 Study Group. Transplantation 1995;59:1285-93.

33. Brewer Y, Palmer A, Taube D, et al. Effect of graft perfusion with two CD45 monoclonal antibodies on incidence of kidney allograft rejection. Lancet 1989;2:935-7. 